\title{
The Implications of the Timing of Diagnosis of Dementia on the Dementia Caregiver
}

\author{
Kenneth K. W. Ng1,2, Melinda Martin-Khan1,3, Maree Farrow ${ }^{4}$, Elizabeth Beattie5, Nancy A. Pachana ${ }^{6}$ \\ ${ }^{1}$ Centre for Research in Geriatric Medicine, Faculty of Medicine, School of Medicine, The University of Queensland, Brisbane, Australia \\ ${ }^{2}$ Queensland Health, Sunshine Coast Hospital \& Health Service, Nambour, Australia \\ ${ }^{3}$ Centre for Online Health, Faculty of Medicine, School of Medicine, The University of Queensland, Brisbane, Australia \\ ${ }^{4}$ Wicking Dementia Research and Education Centre, University of Tasmania, Hobart, Australia \\ ${ }^{5}$ Faculty of Health, School of Nursing, Queensland University of Technology, Brisbane, Australia \\ ${ }^{6}$ School of Psychology, The University of Queensland, Brisbane, Australia \\ Email: kenneth.ng@uq.edu.au
}

How to cite this paper: Ng, K.K.W., Martin-Khan, M., Farrow, M., Beattie, E. and Pachana, N.A. (2016) The Implications of the Timing of Diagnosis of Dementia on the Dementia Caregiver. Advances in Alzheimer's Disease, 5, 143-154.

http://dx.doi.org/10.4236/aad.2016.54010

Received: October 13, 2016

Accepted: November 12, 2016

Published: November 15, 2016

Copyright $\odot 2016$ by authors and Scientific Research Publishing Inc. This work is licensed under the Creative Commons Attribution International License (CC BY 4.0).

http://creativecommons.org/licenses/by/4.0/

\begin{abstract}
This is a consumer-reference feedback and feasibility testing of a protocol to obtain qualitative responses of co-residing caregivers to questions regarding the timing of dementia diagnosis and their experience of the disclosure of a diagnosis of dementia. Data collection involved focus group discussions and individual phone interviews of a convenience sample $(\mathrm{N}=5)$ of an Alzheimer's Australia state based Consumer Advisory Committee. Thematic analysis utilised the Leximancer software. Consumer feedback suggested a reordering of the interview questions and reversing the data collection sequence to reduce the emotional impact on participants. Suggestions were offered to limit the number of participants in the focus group to shorten the duration of the focus group session to prevent fatigue and to provide a support person to improve participant focus group comfort. Responses to the interview questions indicated caregivers retrospectively considered a timely diagnosis would have provided useful dementia-focused planning, reduced the difficulties of living with uncertainty and would have provided more time to obtain information and support. There were strong expectations for medical practitioners to be sensitive to the possibility of dementia and to be cognisant of the diagnostic concerns of caregivers. The diagnosis of dementia and its timing is important to the dementia caregiver in providing an explanation of the problems experienced and allowing earlier organisation of care, future planning and caregiver education to reduce the difficulties of living with undiagnosed and unrecognised dementia.
\end{abstract}

\section{Keywords}

Caregiver, Timing, Diagnosis, Dementia, Co-Residing 


\section{Introduction}

Countries with national dementia strategies are growing [1] [2]. In England, early diagnosis of dementia as an aim of Government policy [3] has been followed up with financial incentives to encourage general practitioners (GPs) to improve early detection of dementia [4]. In Australia, dementia was made the $9^{\text {th }}$ National Health Priority Area by Australian Health Ministers in 2012, and the National Dementia Framework to guide the strategy, released in 2015 [5], includes timely diagnosis as a priority area for action. Timely diagnosis is defined as the period of delay from recognising the symptoms and engaging the health practitioner to actual diagnosis [6].

One benefit of supporting early diagnosis of dementia is that it allows future planning to occur before decision making capacity is impaired [7]. There is a suggestion that timely diagnosis may improve the quality of life of the person with dementia [8] as well as family caregivers [9]. Although caregiving may occur before a formal diagnosis [10], explicitly acknowledged dementia caregiving officially begins with formal diagnosis declared at the diagnostic disclosure appointment. Caregivers may benefit from diagnostic disclosure of dementia by authenticating their need for information and support, and legitimising their access to community and health services and membership of dementia specific organisations [7].

There is scant information relating to the feelings and thoughts of dementia caregivers about the timing of diagnosis. There is also a need to measure the impact of the timing of dementia diagnosis (i.e. diagnosis at early to late stages) on caregiver decision-making behaviour regarding future planning and accessing information and support, as there is a suggestion that earlier access to information, practical and psychosocial support may improve quality of life [11].

In 2012, 2.7 million Australians identified themselves as carers, and 1.9 million of these co-resided in the same households with their care recipients [12]. Around that period, the prevalence of dementia was estimated to be around 298,000 people (with $92 \%$ of these over the age of 65 years) [13] rising to 342,800 in 2015 [14]. It is estimated that $90 \%$ of people with dementia have involvement of carers [13]. Two thirds of dementia caregivers are considered to be co-residing in the same household as the person with dementia [13].

The role of caregiving is challenging as it involves opportunity cost (what the caregiver has to forgo due to the demands of caregiving), burden and increasing responsibilities but is often accepted without question. Time spent caring as the condition progresses has consequences of increased risk of psychological and physical morbidity, plus social and financial disadvantage [15]. Moreover, co-resident caregiving requires additional personal commitment with intimate involvement in the daily challenges posed by cognitive, functional and behavioural/psychological changes of dementia. Non-residing caregivers enjoy better health and have lower objective burden [16] than co-residing caregivers while co-residence is associated with greater lost opportunities and physical burden [17]. Co-residence and longer hours of caregiving are associated with physiological measures of increased stress [18]. 
We are therefore studying the implications of the timing of dementia diagnosis on the dementia caregiver. This paper reports on the testing of the suitability and acceptability of our protocols and interview questions with a small group of consumers. This initial stage involved a consumer reference group that was set up to review and test research protocols by a state base Alzheimer's Association. We also aimed to obtain preliminary issues and concepts elicited by our research questions.

\section{Methods}

Ethical approval was obtained from the University of Queensland Behavioural and Social Sciences Ethical Review Committee. The research is a descriptive qualitative design using a semi-structured interview with open-ended questions with the intended sample being co-residing caregivers of people with dementia. A criterion for inclusion was co-residence by the dementia caregiver with at least 1 year of caregiving (see Table 1 for full criteria). Members of a pre-existing State-based Alzheimer's Australia Consumer Advisory Committee were invited to participate in the project by an investigator of the project (MF). One function of this committee of current and previous caregivers is to test research protocols and questionnaires to reduce unnecessary trauma or stress on consumers/caregivers from poorly designed research material. Verbal consent was obtained from participants prior to provision of the written protocol, and further verbal consent obtained at each distinct research phase. A focus group was held to consider the protocol and questions. The consumers also agreed to trial the research questions as subjects by participating in answering the questions in both a focus group and individual interviews. The reason for using the same interview questions in both individual and focus group settings is because participants may discuss certain issues in individual interviews that they may not feel comfortable sharing in a group situation.

Data were collected using 7 open-ended questions (see Table 2) in focus group interviews (conducted by MF), followed by individual telephone interviews (conducted by LF). Demographic data were only obtained from the individual telephone interviews using the interRAI Caregiver Survey. The focus group duration averaged 62.6 minutes, (Range 61 - 64 mins, S.D. 1.9) and telephone interviews averaged 10.2 minutes (Range 6 - 13 minutes, S.D. 3.6) and were audiotaped, de-identified and transcribed verbatim by the School of Psychology University of Queensland.

\section{Analysis}

Transcripts were loaded into a qualitative analysis computer software program (Leximancer v4.00.22L1, Leximancer manual v4 2011). The programme automatically generated a list of ranked concepts ( 35 word-like and five name-like words). The concept map was generated at intermediate level thematic concepts (the lowest level is where all concepts are considered themes and the highest level is where concepts are coalesced to 1 theme) with the focus group and the individual interviews tagged as two different groups to see if there were any differences in the topics raised by the two different data collecting process (see Figure 1). 
Table 1. Caregiver selection criteria.

\begin{tabular}{cc}
\hline Inclusion Criteria & Exclusion Criteria \\
\hline Co-resides with person with dementia & Non-English speaking caregiver \\
At least one year of caregiving & Caregiver is less than 18 years old \\
Caregiver is 18 years old or over & \\
\hline
\end{tabular}

Table 2. Interview questions for caregiver focus groups and individual caregiver telephone interviews.

Impact of Diagnosis 1 - 3, Timing of Diagnosis 4 - 6, Seeking information/help 7

1) Can you tell me about your feelings when (NAME) was given the official diagnosis of dementia?

2) Can you tell me what issues of concern to you went through your mind at the time (NAME) received the formal diagnosis of dementia?

3) Can you tell me about how you went about doing things after (NAME) received the official diagnosis of dementia?

4) In you opinion/experience do you think there would be positive effects if the dementia was officially diagnosed earlier?

5) In your opinion/experience do you think there would be negative effects if the dementia was officially diagnosed earlier?

6) In your opinion/experience what do you think prevented (if anything did) (NAME) from receiving a formal diagnosis of dementia earlier?

7) How did having an official diagnosis make you feel about approaching people to help you explore groups or organisations for support or for information about dementia or for help with looking after (NAME)?

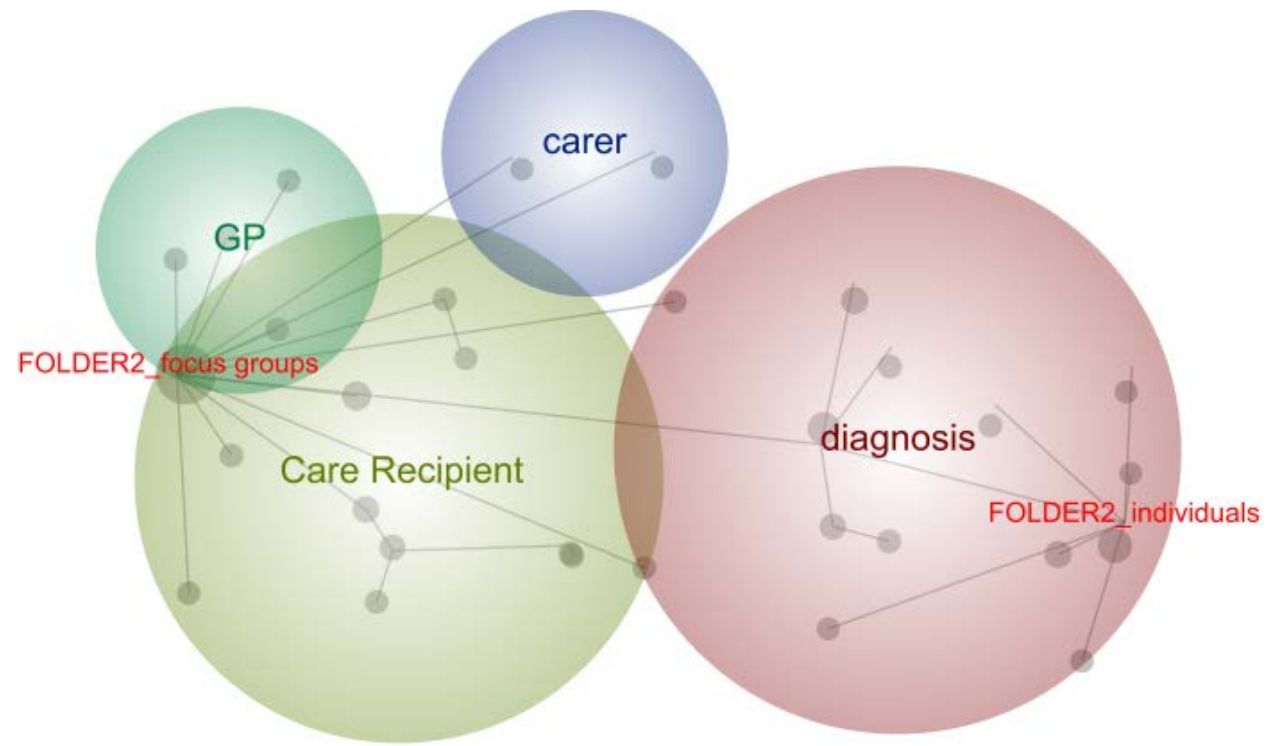

Figure 1. Leximancer concept map of caregivers' themes regarding timing of diagnosis and their experiences at diagnostic disclosure. Technical data: $8 \%$ visibility (labelling concept points), $42 \%$ theme size (size of the circles), 100 degree rotation (degree of vertical or horizontal alignment). 


\section{Results}

\subsection{Demographics}

A total of five individuals (all females) participated-four of the five in focus group discussions and three of the five in individual telephone interviews (one subject only participated in the telephone interview). The relationships of persons with dementia (three females and two males) to the caregiver were three mothers, one husband and one father. All caregivers resided with the person with dementia for at least one year. Demographic data were collected using the interRAI caregiver survey. Detailed demographic data were only collected for subjects who had participated in the individual telephone interviews (three of the five subjects participated in the telephone interviews). All three subjects were university graduates and rated their health as good to excellent (see Table 3 for details).

\subsection{Consumer Feedback}

A change in the sequence of questions was suggested as question one was considered too confronting to begin the interview. A request was made for a support person during focus group meetings in case of emotional upsets. It was considered five persons would be a ideal number for a focus group so as to prevent the session from lasting too long and causing fatigue. The sequence of the individual interview followed by the focus

Table 3. Demographic information from individual telephone interviews (only three caregivers were individually interviewed) (inter-RAI Caregiver Survey).

\begin{tabular}{|c|c|c|c|}
\hline & Participant 1 & Participant 2 & Participant 3 \\
\hline Age (years) & 47 & 59 & 68 \\
\hline Gender M/F & $\mathrm{F}$ & $\mathrm{F}$ & $\mathrm{F}$ \\
\hline Primary Language & English & English & English \\
\hline Level of Schooling & University & University & University \\
\hline Domiciliary Status & Private Home & Private Home & Private Home \\
\hline $\begin{array}{l}\text { Relationship to Person } \\
\text { with Dementia }\end{array}$ & Daughter/Mother & Daughter/Father & Spouse \\
\hline Years as Lived-in Caregiver & 3 & 3 & 7 \\
\hline Self Rating of Own Health & Excellent & Good & Excellent \\
\hline Health Service Use Last 90 days & $1 \mathrm{GP}$ appointment & 3 GP appointments & $1 \mathrm{GP}$ appointment \\
\hline Hearing Difficulty & Normal & $\begin{array}{l}\text { Difficulty in normal } \\
\text { conversation }\end{array}$ & No Difficulty \\
\hline Anhedonia (last 3 days) & Never & Never & Never \\
\hline Anxiety Symptoms (last 3 days) & 1 Day & A few times & Never \\
\hline $\begin{array}{l}\text { Sadness, Depression, } \\
\text { Hopelessness (last } 3 \text { days) }\end{array}$ & Never & Never & Never \\
\hline
\end{tabular}


group meeting was preferred as this may ameliorate the emotional impact of answering questions in a group situation by allowing personal reflection afforded by a preceding individual interview.

\subsection{Thematic Output}

The concept map indicated that the focus group tended towards topics to do with the GP and the person with dementia while the individual interviews tended to discuss issues regarding themselves and the diagnosis. Because of the small numbers, the output from the focus group and the individual interviews were combined for analysis. This offered some confidence that the interview questions evoked issues of relevance to dementia caregivers. Four thematic concepts were generated-Diagnosis; Carer; General Practitioner (GP); Care Recipient (Leximancer had labelled it "Mum" and examination of excerpts revealed this theme included the terms "mum", "father" and "dad", therefore it was considered "care recipient" and relabelled as such). Logs of quotes from the transcripts assigned to these thematic concepts (and combinations of co-occurring concepts within the thematic sphere) were manually read and examined (by $\mathrm{KN}$ ) by constant comparison to gain an understanding of the underlying story of each theme around the factors of the timing of diagnosis and the caregiver. This allowed a researcher led interpretative exercise of discerning contextual settings (the stories) to explain the themes.

\subsubsection{Timing of Diagnosis}

Leximancer thematic concepts of "Diagnosis \& Early": Obtaining an earlier diagnosis allows earlier preparation and information/support seeking to understand the symptoms and needs of the PWD and may avoid reactive decision making and caregiving. A possible higher order theme to explain this would be "smoother care". Excerpts as follows:

Focus Group Caregiver 2: "How would things have been different had she been diagnosed earlier... the really big thing is that all of our decisions were crisis driven... and it could have been so much better for everybody, including my mother, had we been able to do some planning and discussion."

Focus Group Caregiver 4: "If I had been made to understand what the future was I would have done a lot more planning. So, I think the two things you need is early diagnosis crucial, but also the information to back up the diagnosis. Diagnosing some one early and shoving them out the door, there's no point. You have to diagnose them but then make them understand what you' ve diagnosed."

Focus Group Caregiver 1: "I would have liked to have known a lot more earlier and that would have-and-and just from my Mum's perspective, just for her own benefit. We would have given her greater care, ah, quality of care. We wouldn't have been so haphazard in how we were dealing with things I guess."

Individual Interview 1: "She remained without diagnosis, I think, for too long. I think there were signs there that none of us understood or knew what to do with or we did not know how to ask for help to find out what they were." 


\subsubsection{Timing of the Diagnosis and Family Interaction}

Early diagnosis may prolong the negative impact of disagreement with the person with dementia who lacks insight or members of the family in denial. A higher order theme to explain this would be "prolonging conflict". Excerpts as follows:

Individual Interview 2: "Yeah, possibly for my father because as I said, he didn't believe that he had dementia, so it may have impacted him even more negatively. If he was diagnosed earlier."

Individual Interview 3: "and so even if the patient themselves see earlier diagnosis as a negative because they didn't want to know but if - I think for the partners and family, early diagnosis is much more of a positive because of the capacity to plan."

Focus Group Caregiver 2: "because even when it was clear she was quite advanced in not behaving normally there was still rejection of that by-by other members of the family. So I think, you know, had she been more, um-or earlier in her disease stage that would have been even more difficult."

\subsubsection{Diagnosis and Its Impact on Caregiver Actions and Behaviours}

Leximancer thematic concepts of "DIAGNOSIS \& CHANGES": Having a diagnosis triggers a response from the caregiver by crystallising responsibilities and a change of role. The higher order theme to explain this would be "cognitive reframing". Excerpts as follows:

Individual Interview 1: "Yes, I-my behaviour changed immediately after the diagnosis because up till then, I didn't know what was going on with Mum so I was getting very upset and angry with her. The diagnosis put everything in perspective so everymy behaviour towards my mother became, um, how do I describe it... I became more gentle towards her. I showed her more empathy... I didn't yell at her anymore..."

Focus Group Caregiver 1: "I failed to understand why she-these changes were taking place, um, and following diagnosis my behaviour changed. I-I did a complete flip and I was-I deliberately changed my behaviour because I know what she was going through".

Focus Group Caregiver 3: "and I had to make massive changes- he ran a very busy optometry practice, I had to take over as, um, an actual power of attorney".

\subsubsection{Dementia Diagnosis and the Medical Practitioner}

Leximancer thematic concepts of "GP": There is an expectation that medical providers should detect dementia by direct observation and by believing the concerns of the caregiver, and subsequently provide direction to sources of information and support. The higher order theme to explain this would be "engagement". Excerpts as follows:

Focus Group Caregiver 1: "he was a bit dismissive and for somebody in his position not to recognise some of what I was saying-I-I would have liked our GP to have been more aware of what I was saying".

Focus Group Caregiver 3: "he could have had an earlier diagnosis because he clearly had symptoms years before. Yeah, OK. But they were misdiagnosed because of a total lack of knowledge". 
Focus Group Caregiver 1: "if it had been a diagnosis of cancer I would have had every expectation that the GP would have picked it up. So, I think we seem to have a different view of - of, um, dementias... that somehow it's - we have more responsibility with them than the medical community".

Focus Group Caregiver 2: "and if it was me again 10 years ago I would have like the GP to have told me about this organisation. I would have like the specialist who gave us the diagnosis to have told me about this organisation".

Focus Group Caregiver 4: "I think what caused the GP to spring into action was the fact she knew mum before hand, and knew there were definite changes there, she wasn't in any doubt'.

\subsubsection{Support with Information about Dementia}

Leximancer concepts of "DIAGNOSIS \& INFORMATION": The lack of common and general knowledge about dementia delays detection and delays appropriate response after diagnosis. Receiving the dementia diagnosis triggers the caregiver to learn about and understand the condition. The higher order theme to understand this would be "barrier". Excerpts as follows

Focus Group Caregiver 4: "Lack of knowledge on her part to ask the doctor-because it probably took her six months to ask the doctor, so if she had have-or actually I could-I could word it better. If I know more I would have... If I had known more what to look for I would have been a bit more proactive".

Focus Group Caregiver 2: "I think I could say the barrier was I didn't have enough information at the time to be more proactive".

Focus Group Caregiver 3: "Ah, helpful information at diagnosis would have been really usefup'.

Individual Interview 1: “Yeah, I did reach out to Alzheimer's Australia for, ah, information to educate myself about dementia. So I wanted to understand more about what it was".

Focus Group Caregiver 1: "So, you know, like, in all disease situations you're desperate for information on progression".

\section{Discussion}

The testing of the research protocol and open interview questions ascertained the opinions of a consumer-reference group that provided practical and important advice regarding a re-ordering of the research questions and modification to the sequence of data gathering to reduce the emotional effects of the interview. Other changes were suggested to improve the efficiency of the focus group meetings. The research questions explored dementia caregivers' thoughts and feelings at diagnosis, and their opinions regarding its timing. It appears to have elicited useful themes of concern to them.

Diagnosis initiated changes to caregivers' perception of and attitudes and responses to the care recipients, and focused their responsibilities. They considered timely diagnosis could have shortened the period of uncertainty, allowed dementia specific preparation to start at an earlier stage and thereby reduced unfocused care. However, the 
possibility this could result in prolonged contention by disagreements with diagnosis from denial or lack of insight in the person with dementia or family members was acknowledged.

It is recognised that dementia knowledge is important to caregivers [19] and caregivers not knowing enough to ask the appropriate questions have been described in previous studies [20] [21] [22]. This problem is highlighted, in this report, by their regret at a lack of awareness in considering dementia a possibility during its early manifestation, thus delaying detection, and their strong interest after diagnosis to learn to understand and manage it. Some caregivers considered their lack of knowledge and inexperience had hampered effective communication with primary doctors; especially if the doctors were not alert to the possibility of dementia. This was considered to have influenced the timeliness of diagnosis.

The caregivers considered GPs (and subsequently the specialist) as primary sources of diagnostic help and expected them to be sensitive to caregiver concerns. The GP and specialist were also expected to provide guidance to sources of information and support. This is consistent with previous qualitative reports of caregivers at diagnostic disclosure expecting personalised practical advice in addition to generic information [23] [24].

The preliminary data provides some insight into the immediate post-diagnostic dementia caregiver needs (lack of knowledge and confidence, and expectations of support after diagnosis) expressed retrospectively. Admittedly, these responses from experienced caregivers cannot be assumed to apply to caregivers of newly diagnosed people with dementia early in their caregiving trajectory [25]. Additionally, there is little research evidence as to whether the timing of diagnosis (defined as how early in the course of symptomatic dementia when diagnosed [26]) is associated with differences in caregiver needs at diagnosis or in subsequent decisions and actions such as completing legal and health documents.

\section{Limitations}

The interpretation of the analysis should be considered in line with the sample size ( $\mathrm{n}=$ 5 ) and the consenting process (all participants were drawn from a consumer reference group based on their willingness to provide feedback on the protocol). However, the exercise in obtaining consumer-feedback on the protocol provided an opportunity to explore the effectiveness of the open-questions in eliciting issues to do with the timing of diagnosis. Being a member of a consumer reference committee could indicate a bias towards proactivity as reflected by the high education level noted from the individual interviews. This makes generalisation difficult. This did however provide an enriched sample and it is important to consider that this analysis has methodological triangulation (such as interview vs. focus group) [27]. Reflexivity [28] is controlled by critical self reflection and having researchers from the heterogeneity of medical, nursing, psychology, academia and clinical backgrounds. "A priori" biases are unavoidable and Leximancer controls for that in producing the thematic and relational outputs by statisti- 
cally based objective algorithms. Interpretation of the excerpts was still required by the researcher but this was guided by the aims of the research.

\section{Conclusion}

Consumer advice regarding the testing of the research protocol and open-ended questions of the implications of the timing of diagnosis on the dementia caregiver confirms the acceptability of our research protocol and research questions. It is important that consumers have opportunities to contribute as research partners early in the project design stages. Preliminary data from a small and non-representative sample indicate general issues of concern to the dementia caregiver were elicited that appeared to be relevant to our research objective. There are suggestions that the benefits to the caregiver of the care recipient receiving a timely dementia diagnosis include: providing certainty and explanation to allow earlier and smoother dementia specific planning to occur, and the opportunity to seek information about dementia and support. The findings also suggest the caregiver expects the general practitioner and specialist to provide, at the point of diagnosis, direction to resources for information and support. All the caregivers considered that timely diagnosis-directed care and planning informed by knowledge of dementia and its progression could have reduced some of the difficulty they and the care recipients had experienced.

\section{Acknowledgements}

We thank Lilleyane Andrade Figueiredo for performing the telephone interviews. We thank the members of the Alzheimer's Australia Vic Consumer Advisory Committee who participated for contributing their experiences.

\section{Declaration of Conflicting Interests}

The author(s) declared no potential conflicts of interest with respect to the research, authorship, and/or publication of this article.

\section{Funding}

This project was funded by the Dementia Collaborative Research Centre (DCRC) Early Diagnosis and Prevention (EDP).

\section{References}

[1] Alzheimer Europe. National Dementia Plans: A Snapshot of the Status of National Dementia Strategies around Europe. 2013. http://www.alzheimer-europe.org/Policy-in-Practice2/National-Dementia-Plans

[2] Skladzien, E., Bowditch, K. and Rees, G. (2011) National Strategies to Address Dementia: A Report by Alzheimer's Australia. Alzheimer's Australia, Australia.

[3] United Kingdom Department of Health (2009) Living Well with Dementia: A National Dementia Strategy. Department of Health, England.

[4] National Health Service England (2013) Enhanced Service Specification: Facilitating Timely 
Diagnosis and Support for People with Dementia. NHS, England.

[5] Australian Health Ministers Advisory Council (2015) National Framework for Action on Dementia 2015-2019. Department of Social Services, Australia.

[6] De Lepeleire, J., et al. (2008) The Primary Care Diagnosis of Dementia in Europe: An Analysis Using Multidisciplinary, Multinational Expert Groups. Aging \& Mental Health, 12, 568-576. https:/doi.org/10.1080/13607860802343043

[7] Prince, M., Bryce, R. and Ferri, C. (2011) World Alzheimer Report 2011 Benefits of Early Diagnosis and Intervention. Alzheimer's Disease International.

[8] Mate, K.E., et al. (2012) Diagnosis and Disclosure of a Memory Problem Is Associated with Quality of Life in Community Based Older Australians with Dementia. International Psychogeriatrics, 24, 1962-1971. https:/doi.org/10.1017/S1041610212001111

[9] Belle, H., et al. (2006) Ehancing the Quality of Life of Dementia Caregivers from Different Ethic or Racial Groups. Annals of Internal Medicine, 145, 727-738. https:/doi.org/10.7326/0003-4819-145-10-200611210-00005

[10] Gaugler, J.E., Zarit, S.H. and Pearlin, L.I. (2003) The Onset of Dementia Caregiving and Its Longitudinal Implications. Psychology and Aging, 18, 171-180. https:/doi.org/10.1037/0882-7974.18.2.171

[11] de Vugt, M.E. and Verhey, F.R. (2013) The Impact of Early Dementia Diagnosis and Intervention on Informal Caregivers. Progress in Neurobiology, 110, 54-62.

https:/doi.org/10.1016/j.pneurobio.2013.04.005

[12] Australian Bureau of Statistics (2014) Caring in the Community, Australia: Summary of Findings, 2012. ABS Cat. No. 4436.0. Canberra ABS. abs.gov.au/AUSSTATS/abs@.nsf/mf/4436.0

[13] AIHW (2012) Dementia in Australia. Cat. No. Age 70. Australian Institute of Health and Welfare, Canberra.

[14] Australian Institue of Health and Welfare (2015) Dementia. http://www.aihw.gov.au/dementia

[15] Brodaty, H. and Donkin, M. (2009) Family Caregivers of People with Dementia. Dialogues in Clinical Neuroscience, 11, 217-228.

[16] Baiardi, J.M. (1997) The Influence of Health Status, Burden, and Degree of Cognitive Impairment on the Self-Care Agency and Dependent-Care Agency of Caregivers of Elders. Wayne State University, Detroit, 220 p.

[17] Raccichini, A., Castellani, S., Civerchia, P., Fioravanti, P. and Scarpino, O. (2009) The Caregiver's Burden of Alzheimer Patients: Differences between Live-In and Non-Live-In. American Journal of Alzheimer's Disease and Other Dementias, 24, 377-383. http://dx.doi.org/10.1177/1533317509340025

[18] Holland, J.M., et al. (2011) Cortisol Outcomes among Caucasian and Latina/Hispanic Women Caring for a Family Member with Dementia: A Preliminary Examination of Psychosocial Predictors and Effects of a Psychoeducational Intervention. Stress \& Health, 27, 334-346. http://dx.doi.org/10.1002/smi.1375

[19] Stokes, L.A., Combes, H. and Stokes, G. (2014) Understanding the Dementia Diagnosis: The Impact on the Caregiving Experience. Dementia, 13, 59-78. http://dx.doi.org/10.1177/1471301212447157

[20] Abley, C., et al. (2013) Patients' and Carers' Views on Communication and Information Provision When Undergoing Assessments in Memory Services. Journal of Health Services Research \& Policy, 18, 167-173. http://dx.doi.org/10.1177/1355819613479945 
[21] Innes, A., Szymczynska, P. and Stark, C. (2014) Dementia Diagnosis and Post-Diagnostic Support in Scottish Rural Communities: Experiences of People with Dementia and Their Families. Dementia, 13, 233-247. http://dx.doi.org/10.1177/1471301212460608

[22] Manthorpe, J., et al. (2013) From Forgetfulness to Dementia: Clinical and Commissioning Implications of Diagnostic Experiences. British Journal of General Practice, 63, e69-e75. http://dx.doi.org/10.3399/bjgp13x660805

[23] Karnieli-Miller, O., Werner, P., Aharon-Peretz, J., Sinoff, G. and Eidelman, S. (2012) Expectations, Experiences, and Tensions in the Memory Clinic: The Process of Diagnosis Disclosure of Dementia within a Triad. International Psychogeriatrics, 24, 1756-1770. http://dx.doi.org/10.1017/S1041610212000841

[24] Morgan, D.G., et al. (2014) Informal Caregivers' Hopes and Expectations of a Referral to a Memory Clinic. Social Science \& Medicine, 102, 111-118.

http://dx.doi.org/10.1016/j.socscimed.2013.11.023

[25] Boots, L.M.M., Wolfs, C.A.G., Verhey, F.R.J., Kempen, G.I.J.M. and de Vugt, M.E. (2015) Qualitative Study on Needs and Wishes of Early-Stage Dementia Caregivers: The Paradox between Needing and Accepting Help. International Psychogeriatrics, 27, 927-936. http://dx.doi.org/10.1017/S1041610214002804

[26] Brooker, D., La Fontaine, J., Evans, S., Bray, J. and Saad, K. (2014) Public Health Guidance to Facilitate Timely Diagnosis of Dementia: ALzheimer's Cooperative Valuation in Europe Recommendations. International Journal of Geriatric Psychiatry, 29, 682-693. http://dx.doi.org/10.1002/gps.4066

[27] Farmer, T., Robinson, K., Elliott, S.J. and Eyles, J. (2006) Developing and Implementing a Triangulation Protocol for Qualitative Health Research. Qualitative Health Research, 16, 377-394. http://dx.doi.org/10.1177/1049732305285708

[28] Buckner, S. (2005) Taking the Debate on Reflexivity Further. Journal of Social Work Practice, 19, 59-72. http://dx.doi.org/10.1080/02650530500071969

\section{Submit or recommend next manuscript to SCIRP and we will provide best service for you:}

Accepting pre-submission inquiries through Email, Facebook, LinkedIn, Twitter, etc. A wide selection of journals (inclusive of 9 subjects, more than 200 journals)

Providing 24-hour high-quality service

User-friendly online submission system

Fair and swift peer-review system

Efficient typesetting and proofreading procedure

Display of the result of downloads and visits, as well as the number of cited articles

Maximum dissemination of your research work

Submit your manuscript at: http://papersubmission.scirp.org/

Or contact aad@scirp.org 